ÉTUDES Études finno-ougriennes

FINNO-

OUGRIENNES

49-50 | 2018

Travaux de terrain \& varia

\title{
Une noce oudmourte
}

\section{Eva Toulouze}

\section{(2) OpenEdition}

Journals

Édition électronique

URL : https://journals.openedition.org/efo/12337

DOl : $10.4000 /$ efo. 12337

ISSN : 2275-1947

Éditeur

INALCO

Référence électronique

Eva Toulouze, « Une noce oudmourte», Études finno-ougriennes [En ligne], 49-50 | 2018, mis en ligne le 19 février 2019, consulté le 08 juillet 2021. URL : http://journals.openedition.org/efo/12337 ; DOI : https://doi.org/10.4000/efo.12337

Ce document a été généré automatiquement le 8 juillet 2021

\section{cc) (7) (9)}

Études finno-ougriennes est mis à disposition selon les termes de la Licence Creative Commons Attribution - Pas d'Utilisation Commerciale 4.0 International. 


\title{
Une noce oudmourte
}

\author{
Eva Toulouze
}

\section{Introduction}

1 Ce compte rendu de terrain relate une noce oudmourte qui a eu lieu en avril 2018 dans le raïon de Šarkan. J'y ai été invitée non point à titre d'anthropologue mais à titre d'amie de la famille, plus précisément de la mère de la mariée. J'avais en effet fait connaissance avec elle en janvier, car celle-ci est la partenaire scénique de mon ami doctorant oudmourte de l'université de Tartu, Pavel Kutergin. Nous avons sympathisé, j'ai rencontré sa famille, entre autres sa fille, et je ne pouvais pas laisser passer l'occasion de découvrir une noce oudmourte.

Deux remarques préliminaires.

\section{Mariage et concubinage}

3 La société oudmourte traditionnelle a ses valeurs qui persistent en dépit de la pénétration de valeurs nouvelles et des contacts étroits avec la culture russe. Vues d'une perspective occidentale, ce sont des valeurs fort traditionnelles, axées autour de la famille et des liens de parenté. Ceux-ci sont structurants dans la société oudmourte. Autour d'eux s'articule l'entraide quotidienne, par exemple. Les liens à l'intérieur de la grande famille sont renforcés et activés au cours de rituels ad hoc.

Le mariage reste un moment important dans la constitution de ce réseau. Les mariages sont en général stables. Séparations et divorces existent bien entendu, mais ils sont mal vus par la société traditionnelle. Le mariage officiel est l'élément déterminant de l'acquisition d'un nouveau statut. Non point que le concubinage soit exclu. Après les «fiançailles» il est naturel que le couple vive ensemble avant de confirmer leur décision par le mariage - il n'y a pas de tensions autour de la virginité prémaritale comme dans les sociétés traditionnelles catholiques. La question n'est pas là. Le couple de facto n'est pas reconnu comme couple de jure. Le couple de facto n'est pas inscrit dans le réseau de relations familiales, ne confère pas, par exemple, à l'homme les droits d'un 
homme marié - celui d'être sacrificateur par exemple -, ne permet pas l'insertion de la "pièce rapportée " au sein des réseaux de parenté. Donc l'acte du mariage a une importance considérable dans la vie d'une famille.

Cela ne veut pas dire que tous les Oudmourtes se reconnaissent dans ce modèle. Si j'observe le groupe des doctorants de l'université de Tartu, on distingue des approches différentes: un couple (environ 26 ans l'un et l'autre) a fait le choix d'un modèle traditionnel, mariage traditionnel à 20 ans, ils viennent d'avoir leur deuxième enfant ; sur trois jeunes de 28 ans, deux garçons et une fille (tous d'origine rurale), les avis sont partagés : un garçon du sud de l'Estonie se rattache intégralement à la tradition qu'il n'envisage aucunement d'enfreindre, mais il n'est pourtant toujours pas marié. Les trois " traditionalistes ", tout en appréciant une Oudmourte un peu plus âgée qui vit et travaille à Tartu, désapprouvent son choix, qui est de vivre en concubinage avec un Komi, père de ses deux enfants. Ils pensent qu'elle a tort de ne pas se marier. Les deux autres jeunes Oudmourtes, l'un d'un raïon du Nord du pays et l'autre de l'oblast de Kirov envisagent pour eux-mêmes plutôt unconcubinage qu'un mariage traditionnel.

\section{Le raïon de Šarkan}

6 Le raïon de Šarkan présente des traits contradictoires. D'une part c'est l'un des raïons dont la population présente le plus haut pourcentage d'Oudmourtes. L'oudmourte y est encore largement parlé. Mais c'est aussi une région où l'influence quotidienne de la culture russe se fait sentir. Elle est omniprésente : dans le dialecte, où l'abondance des termes issus du russe saute aux yeux; dans les pratiques culturelles, où les habitants du raïon chantent autant de chants russes que de chants oudmourtes. Ainsi, dans ce raïon, par exemple, personne aujourd'hui ne porte le costume oudmourte en dehors de la scène, contrairement à ce qui se passe dans les régions du Sud.

7 Il était donc intéressant de comprendre comment une noce oudmourte a lieu dans ce raïon dans ces conditions, aujourd'hui.

\section{Les circonstances de la noce}

De quelle noce s'agit-il?

9 Le jeune couple, Denis et Maria, a la caractéristique de partager le même nom de famille, Hohrjakov. Ils sont ensemble depuis cinq ans. Ils ont tous deux 23 ans et tous deux travaillent. Denis, issu d'un village éloigné du même raïon, Kivary, relié au monde par une route construite ces dernières années, travaille dans une scierie, où il a des responsabilités. Son père est invalide, et son oncle Aleksej Shkljaev est une célébrité oudmourte, car il a été directeur du quotidien oudmourte Udmurt Dunne. Sa mère est une simple travailleuse agricole.

10 La mariée, Maria, a fait des études d'économie et aide son père, qui est un entrepreneur agricole du bourg de Keldyš assurant la comptabilité de l'entreprise. Elle est résolue à vivre dans un village et à ne pas aller s'installer en ville. Sa famille occupe une position de prééminence dans le village. Si nous étions dans les années 1930, à n'en pas douter, Nikolaj Hohrjakov serait accusé d'être un koulak. En fait, il a construit son entreprise de ses mains et a fait preuve d'un rare esprit d'entreprise. Aujourd'hui, il est 
simplement victime de l'envie environnante, qui a abouti, en janvier 2018, à ce que ses hangars aient été brûlés, détruisant ses équipements... Sa femme, Tatjana Hohrjakova est une personnalité connue aussi bien au niveau du raïon que de la République : elle est par formation professeur d'oudmourte et pratique encore partiellement dans une école du village de Zjuzino. Mais elle est aussi responsable de la maison de la culture du même village et sa réputation est avant tout due au fait qu'elle écrit des poèmes et des chansons, et qu'elle les chante elle-même en s'accompagnant à l'accordéon, en duo avec mon ami Pavel Kutergin (qui est aussi doctorant en ethnologie à l'université de Tartu). Ils ont obtenu beaucoup de reconnaissance à des concours au niveau de la république. C'est ainsi que j'ai été invitée à cette noce : nous nous sommes connues en janvier 2018 et nous avons aussitôt sympathisé.

11 Bien que cela ne ressorte pas clairement de la description que je viens de fournir, il y a un décalage social entre les deux familles, Maria étant issue d'une famille bénéficiant d'un statut reconnu comme supérieur. Cela n'a pas été sans poser de problèmes dans le mariage des jeunes. En effet la famille de Maria était hostile à ce mariage, et seule l'obstination de la jeune fille a réussi à l'imposer. Les objections n'étaient pas uniquement sociales: la famille n'apprécie pas le jeune homme en tant que tel et considère que les jeunes gens ne sont pas au même niveau intellectuel. Mais la grossesse de la mariée a été déterminante pour lever les objections au mariage. Il n'en reste pas moins que c'est la mort dans l'âme que la mère de la mariée a laissé partir sa fille.

12 Les témoignages ethnographiques montrent qu'une noce durait plusieurs jours. $\mathrm{Ce}$ n'est plus le cas aujourd'hui. Mais la tradition de tenir la noce sur deux jours s'est enracinée. Le premier jour est consacré à la famille de la mariée et aux cérémonies officielles. Le deuxième jour à la famille du marié, qui est désormais aussi devenue celle de la mariée.

\section{Le premier jour}

13 Le premier jour commence assez tôt chez la mariée. Au village de Keldyš, la mariée vit dans une très grande maison à étage. Le rez-de-chaussée se compose d'un très grand salon et d'une très grande cuisine, en plus d'une grande véranda. Les chambres, trois chambres spacieuses, en plus d'un atelier, sont à l'étage. Nous sommes accueillis par la mère de la mariée, dont c'est le grand jour. C'est elle qui porte toute la responsabilité de la journée. La mariée a choisi comme témoin une camarade d'université, une fille russe, elle-même responsable d'une petite entreprise. Cela explique que le russe occupe une certaine place dans les activités du matin. Un scénario a été mis au point. L'idée directrice est que le marié vient chercher sa fiancée, accompagné de sa famille et de ses proches, et qu'il faut mettre sur son chemin le plus d'obstacles possibles. Je suis tout de suite enrôlée, ce qui fait que je ne serai pas témoin oculaire des premières étapes de cette chasse au trésor. Je vois, en montant l'escalier, que sur chaque marche sont collées deux ou trois lettres. Le rôle que je dois jouer, c'est celui d'une fausse mariée. En effet, une fois que le marié a réussi à monter l'escalier, il doit identifier sa fiancée. Dans chaque chambre, une fausse fiancée l'attend. Moi, je suis la fausse fiancée venue de l'étranger. On m'affuble de voiles de tulle et je dois rester enfermée dans une des chambres. 

fiancé : les décibels se multiplient aussitôt, et les rires fusent. Il va devoir monter l'escalier : je comprends alors qu'à chaque marche, il doit deviner le sens des lettres qui sont les initiales des tâches qu'il va devoir accomplir une fois marié pour aider sa femme dans la maison. Bien sûr les expressions sont en russe, même si le marié est tout aussi oudmourte que la mariée, mais les personnes qui ont préparé le test ne le sont pas toutes. pratique du comique sur scène, Orina Uri Beri, sont déguisés en diseuses de bonne aventure. Je ne sais pas ce qu'ils prédisent, je n'arrive pas à entendre, et eux, ils ne se retiennent pas pour parler oudmourte. Mais j'entends les rires qui ponctuent leurs phrases.

16 Une fois arrivé sur le palier, quand il ouvre la porte de ma chambre, je me jette à son cou et je professe une grande impatience de son arrivée... Bref, j'improvise à moitié en français à moitié en oudmourte, ce qui fait rire tout le monde. Il me rejette fièrement en déclarant que je ne suis pas sa fiancée... Je saurai plus tard qu'il avait cru avoir affaire à une femme du cru et qu'il a été a posteriori très embarrassé de savoir qui il avait si résolument écarté, et je ne me priverai pas de le lui rappeler par la suite...

Je mets un moment à comprendre ce qui se passe sur le palier, une fois qu'il a été rejoint par sa future épouse... en haut de l'escalier, un homme (le frère du père de Maria), est assis sur un coffre et sur une série d'oreillers. Il faut le déloger pour emporter le coffre, qui contient la dot de la jeune fille. En fait, l'oncle vend les oreillers et le coffre et des enchères s'ensuivent. Mais Denis doit faire attention à ce qu'on ne lui vole pas sa fiancée : à plusieurs reprises, Pavel profite de ce que l'attention du marié se porte sur les enchères pour emporter Maria dans l'une des chambres et l'y enfermer.

Finalement, le coffre est racheté et emporté : la famille du marié le met dans la voiture. Mais tout n'est pas terminé. Tout le monde descend. D'une part, dans le salon, un cercle se forme, qui chante un chant nuptial. Je suppose que c'est le cortège du marié. Tout le monde a l'air de connaître ce chant. D'autre part une collation est offerte dans la cuisine. Nous, en tant que partie du cortège de la mariée, nous ne nous asseyons pas : c'est la famille du marié, les témoins, les personnes actives de part et d'autres qui occupent la table. la laisser partir. Le couple s'assoit sur deux chaises côte à côte, et Tatjana prend une serviette brodée, sur laquelle elle pose un pain maison et, sur le pain, elle pose une icône. Elle va laisser partir sa fille en la bénissant, et elle va placer un moment la serviette avec le pain et l'icône au-dessus de la tête de la jeune fille, avant de lui donner le tout. Tatjana est émue jusqu'aux larmes, mais elle va bravement jusqu'au bout de la cérémonie. La présence de l'icône nous rappelle que nous sommes dans une zone d'Oudmourtes orthodoxes. Pas grand-chose ici ne rappelle l'ancienne religion autochtone.

20 Ainsi s'achève la première partie : tout le monde part, d'abord le cortège du marié, puis nous grignotons quelque chose et nous partons aussi, formant un long cortège. Le soleil est haut dans le ciel, et tout sent le printemps. Toute la neige n'a pas fondu, mais les routes commencent à devenir une mer de boue un peu compliquée pour les voitures... 
21 Nous nous dirigeons tous vers le chef-lieu, le bourg de Šarkan. C'est la maison de la culture qui sert de maison des mariages. Nous y sommes tout particulièrement accueillis par la directrice, Nadežda Volkova, qui est une bonne amie de Pavel et de Tatjana. Elle nous conduit à l'étage, à la grande salle où elle nous a gardé des places à l'avant. C'est mon premier mariage en Russie.

22 La marche nuptiale de Mendelssohn est bien sûr à l'honneur. Mais il y aura aussi d'autres musiques, sans doute canoniques dans ce type de cérémonie en Russie, et méconnues de moi. Un seul reproche, c'est qu'il n'y a pas un seul chant en oudmourte. Or nous sommes dans un raïon largement habité par des Oudmourtes. Et ce n'est pas qu'il n'y ait pas le choix. Sur les thèmes abordés dans la cérémonie, je connais moi-même un choix de chants oudmourtes contemporains qui n'auraient pu qu'embellir la cérémonie.

23 Ce ne sont pas des élus qui officient, mais des fonctionnaires. Sur le côté, des sièges ont été réservés, perpendiculaires aux rangées du public, sur la gauche, pour les parents des mariés; les témoins seront debout avec deux, les encadrant de part et d'autre. Le père du marié n'est pas là, il est gravement malade et redoute les émotions fortes. Le marié attend en face du pupitre, jusqu'à ce que la mariée, toute de blanc vêtue, le rejoigne accompagnée par son père. Au bout d'une courte cérémonie, les mariés doivent échanger les anneaux, s'embrasser et signer. C'est fait, ils sont officiellement mariés, une nouvelle famille est née, c'est ce qui est souligné par tous les discours officiels...

24 Après le départ des mariés, sous un nuage de confettis, il y a du temps libre. Nous en profitons pour nous éloigner un peu de la foule et aller aux portes du domaine du père Noël, Tol Babaj, car aussi bien Pavel qu'Orina veulent fumer. Je leur tiens compagnie quelque peu, surtout que l'endroit est superbe, même si nous n'entrons pas. Le soleil brille, on domine le bourg de Šarkan et les collines environnantes. Ici, les autorités locales ont construit un centre de loisirs tournant autour du personnage du père Noël oudmourte, le Bonhomme Hiver, qui est l'un des centres du père Noël en Russie et dans le monde. Le père Noël y accueille les enfants, un centre d'artisanat y offre des objets fabriqués par des artisans expérimentés, et des espaces de sport et de loisir sont là pour faire passer le temps. Les locaux et d'autres yorganisent des fêtes, des anniversaires... Mais là, nous nous contentons d'apprécier le paysage et de nous reposer un peu de la masse humaine qui nous attend.

Pendant ce temps, les mariés et les jeunes sont partis, en voiture, faire un tour dans la campagne. Ils doivent passer au moins par un pont, que le marié franchit en portant sa femme, et auquel ils attacheront un loquet dont la clé sera jetée dans la rivière. C'est un moment, pour eux de détente avec leurs amis, sans la contrainte de la présence des personnes plus âgées. Nous, nous retournons bientôt au bourg et nous rejoignons Tatjana, qui prépare une cantine à accueillir les 120 invités de la noce. Là aussi, c'est sa responsabilité. Mais sa fille a pris sur elle l'organisation (anticipée) des décors. Elle a voulu un décor bleu et jaune, et ces couleurs de retrouveront dans tous les éléments de décoration : nappes, napperons (en papier), ballons. Petit à petit les tables se couvrent de mets froids et de bouteilles, en attendant les invités. Nous bavardons en coulisses, Tatjana et Pavel répètent un ou deux chants qu'ils vont présenter, l'animatrice arrive et nous discutons avec elle.

Oksana Bisar est une poétesse et une personnalité de la scène oudmourte. Militante de la langue, elle anime notamment des discothèques oudmourtes. C'est une femme très 
vive dans les quarante-cinq ans, et j'ai eu l'occasion de la connaitre justement dans une de ces soirées discothèque conçues pour les plus de trente ans (mais où tous participent). Elle fait tout en oudmourte, chante elle-même, anime des jeux et transmet une énergie considérable. Cette fois-ci, elle est un peu déçue. On lui a demandé une animation bilingue. Elle m'avoue qu'elle préfère les animations exclusivement en oudmourte... Cela veut dire que la musique sera elle aussi mixte, russe et oudmourte. La situation est typique. Dans le public, il y a peut-être dix personnes qui ne parlent pas oudmourte... Et je ne me compte pas, car j'apprécie pour ma part quand c'est la langue locale qui domine. Nous sommes d'ailleurs les deux seules à porter une robe oudmourte. Cette tradition, dans ce raïon, s'est perdue.

Pour apprécier cette soirée, je suis en difficulté. Je n'ai jamais assisté à une noce en Russie. J'ignore donc ce qui, dans les animations qu'elle offre, est canonique, ce qui est personnel, et ce qui est oudmourte. Elle va proposer différents petits rituels qui, je suppose, n'ont pas grand-chose de traditionnel.

Elle accueille, vers dix-sept heures, les invités et les mariés devant la porte. La mère du marié a des petits pâtés à la viande sur une serviette, qu'elle leur offre avec deux verres de lait. Les deux mères tiennent la serviette bien haut et le couple passe dessous. Oksana a aussi distribué des rubans, bleus et blancs, pour distinguer la compagnie de la mariée et celle du marié. Les neutres, comme elle, porteront un ruban jaune.

Dans la salle, les mariés et leurs parents occupent la table centrale. Les groupes de l'un et de l'autre se font connaître. Chacun se lève, chante, forme une équipe qui, dans les jeux qui auront lieu tout au long de la soirée, se confrontera amicalement à l'autre. Une manière de faire connaissance. La mère du fiancé est un peu tendue. Tout le monde se contrôle, et on constatera à la fin qu'il n'y a pas - chose très rare - de viande soûle. Il n'y a pas réellement de fusion non plus entre les groupes, qui restent bien entre eux, avec des tables entières occupées par la famille et les amis de l'un ou de l'autre des époux.

Oksana est excellente. Elle est en permanence sur le devant de la scène, et pourtant elle ne fatigue pas son auditoire. Les mariés sont souvent sollicités. Le marié reçoit même, dans un jeu, une tarte à la crème à la figure... Ils sont souvent sollicités pour s'embrasser. C'est là une pratique largement répandue en Russie et autour de la Russie : la compagnie crie "Amer» (russe : горько!; estonien : Kibe!; oudmourte : курыт!) et là-dessus les mariés doivent se lever et s'embrasser. On se demande s'ils n'épuisent pas leur arsenal de baisers pour la vie... Maria, enceinte de 6 mois, avouera qu'elle fatigue...

31 La soirée se termine vers minuit. Curieusement la famille du marié et (moins curieusement) les mariés partent vite. Cela laisse la famille de la mariée avec la responsabilité de tout ranger. La partie de la mariée va continuer la fête chez Tatjana et Nikolaj. Le père de famille va vite dormir. Mais les sœurs de Tatjana et leurs maris se rassemblent dans la cuisine, avec le témoin de la mariée et notre groupe à nous. La conversation durera jusqu'à trois heures du matin au moins, avec des moments un peu difficiles de "règlements de compte » entre sœurs. Ceci pour rappeler que même là où les liens familiaux sont d'une importance première dans l'identification sociale de la personne tout n'est pas toujours simple, et Pavel a beau jeu d'opposer l'harmonie de ses relations avec ses trois frères - «ce seront toujours les premiers sur lesquels je peux compter»-et les tensions (somme toute) assez légères, qui traversent les relations entre les sœurs de Tatjana. Il est prévu que nous dormions au même endroit. 


\section{Le deuxième jour} produits alimentaires (ce qui n'empêche pas les invités de chanter les louanges de la nouvelle maîtresse de maison. Mais là, sans doute en raison de l'exiguïté de la cuisine, les choses se sont passées sans encombre. La deuxième épreuve de la mariée, c'est le balayage du salon. Là, en revanche, tout le monde a essayé de l'empêcher de mener sa tâche à bien. Qui apportait de l'extérieur de la paille, qui jetait par terre des pièces de monnaie, qui recouvrait les parties déjà nettoyées. Ce sont les enfants qui, plus que tous, s'amusent : ils rampent parmi les jambes pour mettre la main sur les pièces de monnaie.

La mariée est partie passer la nuit chez son époux. Ils n'ont pas encore de domicile propre, qui sera prêt pendant l'été, et donc elle est amenée à vivre avec sa belle-mère. Le groupe du côté de sa famille à elle va donc poursuivre la noce chez le marié. C'est ce que nous faisons. Nous partons, vers onze heures (après quelques coups de téléphone pour presser notre arrivée), et nous nous rendons dans le village de Kivary. Par la rivière, il est directement relié au village de Pavel, Karsašur, mais par la route c'est compliqué d'y arriver.

plusieurs voitures, et nous y sommes accueillis somptueusement, avec de l'alcool maison. La maison, la cuisine, tout est beaucoup plus petit (beaucoup plus ordinaire) que chez Tatjana. Mais on trouvera de la place pour tout le monde. La table est mise. Dans d'autres régions d'Oudmourtie, notamment au sud, cette deuxième journée est vécue totalement à l'oudmourte, et la mariée elle-même est habillée avec une robe oudmourte. Là, Maria porte une robe ordinaire et personne ne porte d'habits oudmourtes.

son beau-frère porte des toasts. C'est une fête traditionnelle, on chante un peu, même si la place pour danser est limitée. Mais tout le monde attend les cérémonies du jour. des taban' (crêpes à la pâte levée) qu'elle a préparées elle-même. La cuisine est petite, peu arrivent à y pénétrer. En fait, la pâte a été faite par sa belle-mère. Dans la cuisine, il y a un poêle traditionnel, Maria le met à chauffer et sa belle-mère fait les premières crêpes. Puis ce sera son tour, et elle les apportera dans la salle où les invités s'arracheront les crêpes de la mariée, qui témoignent de son savoir-faire de ménagère. À noter que dans la région de Šarkan, les crêpes traditionnelles sont petites, et il en rentre plusieurs dans une poêle, à la différence du sud, où les taban' couvrent toute la surface de la poêle.

En fait, traditionnellement, les invités essayent de lui mettre des bâtons dans les roues, de sorte que les crêpes qui arrivent sur la table sont souvent couvertes d'autres Et puis vient le moment tant attendu, la dernière tâche de la mère de la mariée. On ouvre le coffre et on en exhibe le contenu. J'ai vu d'anciens coffres avec les merveilles de travaux manuels qu'ils contenaient... Jadis, c'était le trousseau de la fiancée, les choses qu'elle avait confectionnées elle-même, qui étaient exhibées à ce moment du rituel. Les choses ont bien changé. Le coffre ne contient rien de traditionnel. Il contient de la literie, dans des sacs en plastique. Des draps, des housses de couette, des couettes, 
des couvertures. Il s'agit plutôt d'un déploiement de richesse que de beauté. Tout est ouvert et exposé, et puis remis dans le coffre.

La tâche de Tatjana est achevée. Sa fille est mariée, elle n'a plus de fonction dans la noce, et elle pourrait se relâcher et faire la fête. Mais la tension a été trop grande et elle craque. Il faut vite l'amener à l'hôpital, et, rompant avec la tradition qui veut que les mariés restent à la fête jusqu'au bout, c'est son gendre qui l'emmène, car son mari a bu... Elle sera ramenée à la maison et Denis reviendra et profitera de sa fête jusqu'au bout.

Tout continue maintenant comme une fête oudmourte ordinaire avec boisson, chants et danses. À un moment, le petit verre est rempli par terre, et les volontaires doivent le boire sans le toucher de leurs mains : il s'agit de le prendre dans sa bouche et d'y renverser le contenu. Hommes et femmes font des démonstrations fort convaincantes. Les danses sont endiablées. Parmi les amis des mariés il se trouve des jeunes gens très bons danseurs, qui se livrent complètement. La place est limitée, les plus timides s'abstiennent.

Petit à petit les gens partent, Pavel sort son accordéon, les jeunes gens vont au sauna, et encore longtemps ils chanteront et danseront dans la rue. Indiscutablement, quand on repartira, l'alcool se fera sentir, mais toujours de manière très correcte. Les compagnies sont joyeuses et curieuses, il est agréable de discuter avec eux. Le responsable des festivités est le frère du maitre de maison (qui ne fera qu'une apparition quand il ne restera plus que peu d'invités, et se plaindra de ce que les jeunes aient bu), qui est très excité de ma présence (puisqu'en pays oudmourte, surtout parmi les intellectuels, mon nom est assez connu). Il ne prononcera pas moins de cinq toasts en mon honneur, ce qui m'amènera à essayer de l'éviter pendant toute la fin de la soirée.

\section{Conclusion}

Un mariage, somme toute, réussi. Toutes les tensions ne sont pas levées entre les deux parties, mais le jeune couple est confirmé et attend avec impatience son premier enfant. Denis a essayé de nouer des liens avec les jeunes de l'entourage de Tatjana, qui l'acceptent nettement mieux que sa belle-mère, au moins pour l'instant. Les adultes sont contents de la manière tranquille dont les choses se sont déroulées. Notre présence, quelque curieux que cela paraisse, a conféré à la noce un certain statut social, qui a été apprécié par toutes les parties. J'ai sans doute un peu de mal à en comprendre les tenants et les aboutissants, mais je dois me plier aux résultats de mes observations. Pour moi, une première expérience, bien enracinée localement, qui, je l'espère, me servira de base dans l'appréciation des occasions suivantes qui ne manqueront pas de se présenter, puisque je suis entourée de jeunes Oudmourtes qui voient leur avenir dans un mariage traditionnel... 
INDEX

motscleset pulmad, antropoloogia, rahvaluule, traditsiooniline kultuur, 21 sajand algus, Udmurtid, udmurdi, vene, Eesti, Karsašur, Keldõš, Kirov, Kivarõ, Udmurdimaa, Sharkan, Zjuzino, Tartu, Venemaa

Mots-clés : noce, noce, anthropologie, anthropologie, oralité, oralité, culture traditionnelle, culture traditionnelle, XXIe siècle début, XXIe siècle début, Oudmourtes, Oudmourtes, oudmourte, oudmourte, russe, russe, Estonie, Estonie, Karsašur, Karsašur, Keldyš, Keldyš, Kirov, Kirov, Kivary, Kivary, Oudmourtie, Oudmourtie, Russie, Russie, Šarkan, Šarkan, Tartu, Tartu, Zjuzino, Zjuzino

Keywords : wedding, anthropology, Folklore, Traditional culture, 21th century beginning, Udmurt, Russian, Estonia, Karsashur, Keldysh, Kirov, Kivary, Russia, Sharkan, Tartu, Udmurtia, Zyuzino 